PROCEEDINGS OF THE

AMERICAN MATHEMATICAL SOCIETY

Volume 131, Number 2, Pages 449-453

S 0002-9939(02)06544- 9

Article electronically published on May 17, 2002

\title{
EXPOSED 2-HOMOGENEOUS POLYNOMIALS ON HILBERT SPACES
}

\author{
SUNG GUEN KIM AND SANG HUN LEE
}

(Communicated by Jonathan M. Borwein)

\begin{abstract}
We show that every extreme point of the unit ball of 2-homogeneous polynomials on a separable real Hilbert space is its exposed point and that the unit ball of 2-homogeneous polynomials on a non-separable real Hilbert space contains no exposed points. We also show that the unit ball of 2homogeneous polynomials on any infinite dimensional real Hilbert space contains no strongly exposed points.
\end{abstract}

We recall that a unit vector $x$ in a real Banach space $E$ is exposed if there is a unit vector $f \in E^{*}$ so that $f(x)=1$ and $f(y)<1$ for each $y \in B_{E}$ with $y \neq x$, where $B_{E}$ is the closed unit ball of $E$. It is easy to see that every exposed point of $B_{E}$ is an extreme point. A unit vector $x$ in a real Banach space $E$ is strongly exposed if there is a unit vector $f \in E^{*}$ so that $f(x)=1$ and given any sequence $\left(x_{k}\right)$ in $B_{E}$ with $f\left(x_{k}\right) \rightarrow 1$ we can conclude that $x_{k} \rightarrow x$ in norm. It is easy to see that every strongly exposed point of $B_{E}$ is its exposed point. We denote by $\operatorname{ext} B_{E}, \exp B_{E}$ and $\operatorname{sexp} B_{E}$ the sets of extreme points, exposed points and strongly exposed points of $B_{E}$, respectively.

Let $\mathcal{P}\left({ }^{2} H\right)$ be the Banach space of continuous 2-homogeneous polynomials on a real Hilbert space $H$. Recently many authors (see [1- 7]) studied extremal problems for polynomials on a Banach space. The extreme points of the unit ball of this space has been characterized by Grecu [6]. The object of this note is to determine the exposed and strongly exposed points of the unit ball of $\mathcal{P}\left({ }^{2} H\right)$. Grecu showed that for a real Hilbert space $H, P \in \operatorname{ext} B_{\mathcal{P}\left({ }^{2} H\right)}$ if and only if there exists an orthogonal decomposition of $H=H_{1} \oplus H_{2}$ such that $P(x)=\left\|\pi_{1}(x)\right\|^{2}-\left\|\pi_{2}(x)\right\|^{2}$, where $\pi_{j}: H \rightarrow H_{j}$ are the orthogonal projections of $H$ onto $H_{j} \quad(j=1,2)$. Using this result we show the following results:

(1) If $H$ is a separable real Hilbert space, then every extreme point of the unit ball of $\mathcal{P}\left({ }^{2} H\right)$ is exposed.

(2) If $H$ is a non-separable real Hilbert space, then the unit ball of $\mathcal{P}\left({ }^{2} H\right)$ contains no exposed points.

(3) If $H$ is an infinite dimensional real Hilbert space, then the unit ball of $\mathcal{P}\left({ }^{2} H\right)$ contains no strongly exposed points.

Received by the editors January 15, 2001 and, in revised form, September 10, 2001.

2000 Mathematics Subject Classification. Primary 46B20, 46E15.

The first author wishes to acknowledge the financial support of the Korea Research Foundation (KRF-2000-015-DP0012).

The second author wishes to acknowledge the financial support by KOSEF research No. (20011-10100-007).

(C)2002 American Mathematical Society 
Theorem 1. Let $H$ be a separable real Hilbert space. Then every extreme point of the unit ball of $\mathcal{P}\left({ }^{2} H\right)$ is exposed.

Proof. Since $\exp B_{\mathcal{P}\left({ }^{2} H\right)} \subset \operatorname{ext} B_{\mathcal{P}\left({ }^{2} H\right)}$, it suffices to show that if $P \in \operatorname{ext} B_{\mathcal{P}\left({ }^{2} H\right)}$, then $P \in \exp B_{\mathcal{P}\left({ }^{2} H\right)}$.

Let $P \in \operatorname{ext} B_{\mathcal{P}\left({ }^{2} H\right)}$. By Theorem 1.6 of $\left[\underline{6}, P(x)=\left\|\pi_{1}(x)\right\|^{2}-\left\|\pi_{2}(x)\right\|^{2}\right.$ where $H=H_{1} \oplus H_{2}$ and $\pi_{j}: H \rightarrow H_{j}$ are the orthogonal projections of $H$ onto $H_{j} \quad(j=$ $1,2)$. Clearly $\left\|\pi_{j}\right\|=1$. Let $\left\{e_{\alpha}\right\}_{\alpha \in A}$ and $\left\{t_{\beta}\right\}_{\beta \in B}$ be orthonormal bases of $H_{1}$ and $H_{2}$, respectively. It is clear that $\left\{e_{\alpha}, t_{\beta}\right\}_{\alpha \in A, \beta \in B}$ is an orthonormal basis of $H$. Then for each $x \in H$, we have

$$
x=\sum_{\alpha \in A}\left\langle x, e_{\alpha}\right\rangle e_{\alpha}+\sum_{\beta \in B}\left\langle x, t_{\beta}\right\rangle t_{\beta}
$$

and

$$
P(x)=\sum_{\alpha \in A}\left\langle x, e_{\alpha}\right\rangle^{2}-\sum_{\beta \in B}\left\langle x, t_{\beta}\right\rangle^{2} .
$$

Note that $P\left(e_{\alpha}\right)=1$ for all $\alpha \in A$ and $P\left(t_{\beta}\right)=-1$ for all $\beta \in B$. Let $\left\{a_{\alpha}\right\}_{\alpha \in A}$ and $\left\{b_{\beta}\right\}_{\beta \in B}$ be collections of reals such that $a_{\alpha}>0, b_{\beta}<0$ and

$$
\sum_{\alpha \in A} a_{\alpha}-\sum_{\beta \in B} b_{\beta}=1
$$

Define $f \in \mathcal{P}\left({ }^{2} H\right)^{*}$ such that for each $Q \in \mathcal{P}\left({ }^{2} H\right)$,

$$
f(Q)=\sum_{\alpha \in A} Q\left(e_{\alpha}\right) a_{\alpha}+\sum_{\beta \in B} Q\left(t_{\beta}\right) b_{\beta} .
$$

Then $\|f\|=1$. Indeed, for each $Q \in \mathcal{P}\left({ }^{2} H\right)$ with $\|Q\|=1$, we have $\left|Q\left(e_{\alpha}\right)\right| \leq 1$ for all $\alpha \in A,\left|Q\left(t_{\beta}\right)\right| \leq 1$ for all $\beta \in B$ and

$$
|f(Q)| \leq \sum_{\alpha \in A}\left|Q\left(e_{\alpha}\right)\right| a_{\alpha}+\sum_{\beta \in B}\left|Q\left(t_{\beta}\right)\right|\left(-b_{\beta}\right) \leq \sum_{\alpha \in A} 1 \cdot a_{\alpha}-\sum_{1 \cdot \beta \in B} b_{\beta}=1
$$

and

$$
f(P)=\sum_{\alpha \in A} P\left(e_{\alpha}\right) a_{\alpha}+\sum_{\beta \in B} P\left(t_{\beta}\right) b_{\beta}=\sum_{\alpha \in A} 1 \cdot a_{\alpha}-\sum_{\beta \in B} 1 \cdot b_{\beta}=1 .
$$

We will show that this functional $f$ exposes the polynomial $P$.

Let $Q \in \mathcal{P}\left({ }^{2} H\right)$ be such that $f(Q)=1=\|Q\|$. We claim that $Q\left(e_{\alpha}\right)=1$ for all $\alpha \in A$ and $Q\left(t_{\beta}\right)=-1$ for all $\beta \in B$. Indeed,

$$
1=f(Q)=\sum_{\alpha \in A} Q\left(e_{\alpha}\right) a_{\alpha}+\sum_{\beta \in B} Q\left(t_{\beta}\right) b_{\beta}=\sum_{\alpha \in A} 1 \cdot a_{\alpha}-\sum_{\beta \in B} 1 \cdot b_{\beta}=1,
$$

so $Q\left(e_{\alpha}\right)=1$ for all $\alpha \in A$ and $Q\left(t_{\beta}\right)=-1$ for all $\beta \in B$ because of $a_{\alpha}>0, b_{\beta}<0$.

Let $\hat{Q}$ be the corresponding continuous symmetric bilinear form to the polynomial $Q$.

We claim:

(1) $\hat{Q}\left(e_{\alpha}, e_{\alpha^{\prime}}\right)=0 \quad\left(\alpha \neq \alpha^{\prime} \in A\right)$;

(2) $\hat{Q}\left(t_{\beta}, t_{\beta^{\prime}}\right)=0 \quad\left(\beta \neq \beta^{\prime} \in B\right)$;

(3) $\hat{Q}\left(e_{\alpha}, t_{\beta}\right)=0 \quad(\alpha \in A, \beta \in B)$. 
Proof of (1). It follows that

$$
\begin{aligned}
1=\|Q\| & \geq \sup _{x_{\alpha}^{2}+x_{\alpha^{\prime}}^{2}=1}\left|Q\left(x_{\alpha} e_{\alpha}+x_{\alpha^{\prime}} e_{\alpha^{\prime}}\right)\right| \\
& =\sup _{x_{\alpha}^{2}+x_{\alpha^{\prime}}^{2}=1}\left|\hat{Q}\left(x_{\alpha} e_{\alpha}+x_{\alpha^{\prime}} e_{\alpha^{\prime}}, x_{\alpha} e_{\alpha}+x_{\alpha^{\prime}} e_{\alpha^{\prime}}\right)\right| \\
& =\sup _{x_{\alpha}^{2}+x_{\alpha^{\prime}}^{2}=1}\left|Q\left(e_{\alpha}\right) x_{\alpha}^{2}+Q\left(e_{\alpha^{\prime}}\right) x_{\alpha^{\prime}}^{2}+2 \hat{Q}\left(e_{\alpha}, e_{\alpha^{\prime}}\right) x_{\alpha} x_{\alpha^{\prime}}\right| \\
& =\sup _{x_{\alpha}^{2}+x_{\alpha^{\prime}}^{2}=1}\left|x_{\alpha}^{2}+x_{\alpha^{\prime}}^{2}+2 \hat{Q}\left(e_{\alpha}, e_{\alpha^{\prime}}\right) x_{\alpha} x_{\alpha^{\prime}}\right| \\
& =\sup _{x_{\alpha}^{2}+x_{\alpha^{\prime}}^{2}=1}\left|1+2 \hat{Q}\left(e_{\alpha}, e_{\alpha^{\prime}}\right) x_{\alpha} x_{\alpha^{\prime}}\right|
\end{aligned}
$$

which implies $\hat{Q}\left(e_{\alpha}, e_{\alpha^{\prime}}\right)=0$.

Proof of (2). By the similar proof of (1), we have

$$
1=\|Q\| \geq \sup _{x_{\beta}^{2}+x_{\beta^{\prime}}^{2}=1}\left|Q\left(x_{\beta} t_{\beta}+x_{\beta^{\prime}} t_{\beta^{\prime}}\right)\right|=\sup _{x_{\beta}^{2}+x_{\beta^{\prime}}^{2}=1}\left|1+2 \hat{Q}\left(t_{\beta}, t_{\beta^{\prime}}\right) x_{\beta} x_{\beta^{\prime}}\right|
$$

which implies $\hat{Q}\left(t_{\beta}, t_{\beta^{\prime}}\right)=0$.

Proof of (3). By the similar proof of (1), we have

$$
\begin{aligned}
1 & =\|Q\| \geq \sup _{x_{\alpha}^{2}+x_{\beta}^{2}=1}\left|Q\left(x_{\alpha} e_{\alpha}+x_{\beta} t_{\beta}\right)\right| \\
& =\sup _{x_{\alpha}^{2}+x_{\beta}^{2}=1}\left|Q\left(e_{\alpha}\right) x_{\alpha}^{2}+Q\left(t_{\beta}\right) x_{\beta}^{2}+2 \hat{Q}\left(e_{\alpha}, t_{\beta}\right) x_{\alpha} x_{\beta}\right| \\
& =\sup _{x_{\alpha}^{2}+x_{\beta}^{2}=1}\left|x_{\alpha}^{2}-x_{\beta}^{2}+2 \hat{Q}\left(e_{\alpha}, t_{\beta}\right) x_{\alpha} x_{\beta}\right| .
\end{aligned}
$$

By Lemma 2.1 of [3], we have $\hat{Q}\left(e_{\alpha}, t_{\beta}\right)=0$. For $x \in H$, it follows that

$$
\begin{aligned}
Q(x)= & \hat{Q}(x, x) \\
= & \hat{Q}\left(\sum_{\alpha \in A}\left\langle x, e_{\alpha}\right\rangle e_{\alpha}+\sum_{\beta \in B}\left\langle x, t_{\beta}\right\rangle t_{\beta}, \sum_{\alpha \in A}\left\langle x, e_{\alpha}\right\rangle e_{\alpha}+\sum_{\beta \in B}\left\langle x, t_{\beta}\right\rangle t_{\beta}\right) \\
= & \sum_{\alpha, \alpha^{\prime} \in A}\left\langle x, e_{\alpha}\right\rangle\left\langle x, e_{\alpha^{\prime}}\right\rangle \hat{Q}\left(e_{\alpha}, e_{\alpha^{\prime}}\right)+\sum_{\beta, \beta^{\prime} \in B}\left\langle x, t_{\beta}\right\rangle\left\langle x, t_{\beta^{\prime}}\right\rangle \hat{Q}\left(t_{\beta}, t_{\beta^{\prime}}\right) \\
& \quad+2 \sum_{\alpha \in A, \beta \in B}\left\langle x, e_{\alpha}\right\rangle\left\langle x, t_{\beta}\right\rangle \hat{Q}\left(e_{\alpha}, t_{\beta}\right) \\
= & \sum_{\alpha \in A} Q\left(e_{\alpha}\right)\left\langle x, e_{\alpha}\right\rangle^{2}+\sum_{\beta \in B} Q\left(t_{\beta}\right)\left\langle x, t_{\beta}\right\rangle^{2} \quad \text { (by claims (1)-(3)) } \\
= & \sum_{\alpha \in A}\left\langle x, e_{\alpha}\right\rangle^{2}-\sum_{\beta \in B}\left\langle x, t_{\beta}\right\rangle^{2}=P(x),
\end{aligned}
$$

showing that $f$ exposes $P$. Therefore $P \in \exp B_{\mathcal{P}\left({ }^{2} H\right)}$.

Theorem 2. Let $H$ be an infinite dimensional real Hilbert space. Then the unit ball of $\mathcal{P}\left({ }^{2} H\right)$ contains no strongly exposed points.

Proof. Since $\operatorname{sexp} B_{\mathcal{P}\left({ }^{2} H\right)} \subset \operatorname{ext} B_{\mathcal{P}\left({ }^{2} H\right)}$, it suffices to show that

$$
\operatorname{sexp} B_{\mathcal{P}\left({ }^{2} H\right)} \cap \operatorname{ext} B_{\mathcal{P}\left({ }^{2} H\right)}=\emptyset .
$$


Let $P \in \operatorname{ext} B_{\mathcal{P}\left({ }^{2} H\right)}$. By Theorem 1.6 of [6] $P(x)=\left\|\pi_{1}(x)\right\|^{2}-\left\|\pi_{2}(x)\right\|^{2}$ where $H=H_{1} \oplus H_{2}$ and $\pi_{j}: H \rightarrow H_{j}$ are the orthogonal projections of $H$ onto $H_{j}$ $(j=1,2)$. Clearly $\left\|\pi_{j}\right\|=1$. Without loss of generality, assume that $\operatorname{dim}\left(H_{1}\right)=\infty$. Let $\left\{e_{\alpha}\right\}_{\alpha \in A}$ be an orthonormal basis of $H_{1}$. Let $\left\{\alpha_{j}\right\}_{j=1}^{\infty} \subset A$. It is clear that for each $x \in H$, we have $\pi_{1}(x)=\sum_{\alpha \in A}\left\langle\pi_{1}(x), e_{\alpha}\right\rangle e_{\alpha}$. Then

$$
P(x)=\sum_{\alpha \in A}\left\langle\pi_{1}(x), e_{\alpha}\right\rangle^{2}-\left\|\pi_{2}(x)\right\|^{2} .
$$

Suppose that $P \in \operatorname{sexp} B_{\mathcal{P}\left({ }^{2} H\right)}$. Then there is an $f \in \mathcal{P}\left({ }^{2} H\right)^{*}$ such that $\|f\|=1=$ $f(P)$ and given any sequence $\left\{P_{j}\right\}$ in $B_{\mathcal{P}\left({ }^{2} H\right)}$ with $f\left(P_{j}\right) \rightarrow 1$, we have $\left\|P_{j}-P\right\| \rightarrow 0$. For each $\alpha_{j}$, we have

$$
\begin{aligned}
& f\left(\sum_{\alpha \neq \alpha_{j}}\left\langle\pi_{1}(x), e_{\alpha}\right\rangle^{2}-\left\langle\pi_{1}(x), e_{\alpha_{j}}\right\rangle^{2}-\left\|\pi_{2}(x)\right\|^{2}\right)<1 \\
= & f\left(\sum_{\alpha \in A}\left\langle\pi_{1}(x), e_{\alpha}\right\rangle^{2}-\left\|\pi_{2}(x)\right\|^{2}\right),
\end{aligned}
$$

so $f\left(\left\langle\pi_{1}(x), e_{\alpha_{j}}\right\rangle^{2}\right)>0$. We will show $f\left(\left\langle\pi_{1}(x), e_{\alpha_{j}}\right\rangle^{2}\right) \rightarrow 0$ as $j \rightarrow \infty$. For each $n$,

$$
\begin{aligned}
& \sum_{1 \leq j \leq n} f\left(\left\langle\pi_{1}(x), e_{\alpha_{j}}\right\rangle^{2}\right)=f\left(\sum_{1 \leq j \leq n}\left\langle\pi_{1}(x), e_{\alpha_{j}}\right\rangle^{2}\right) \\
\leq & \|f\|\left\|\sum_{1 \leq j \leq n}\left\langle\pi_{1}(x), e_{\alpha_{j}}\right\rangle^{2}\right\|=\left\|\sum_{1 \leq j \leq n}\left\langle\pi_{1}(x), e_{\alpha_{j}}\right\rangle^{2}\right\| \\
= & \sup _{\|x\|=1} \sum_{1 \leq j \leq n}\left\langle\pi_{1}(x), e_{\alpha_{j}}\right\rangle^{2}=\sup _{\|x\|=1}\left\|\pi_{1}(x)\right\|^{2}=\left\|\pi_{1}\right\|^{2}=1 .
\end{aligned}
$$

Thus $\sum_{1 \leq j<\infty} f\left(\left\langle\pi_{1}(x), e_{\alpha_{j}}\right\rangle^{2}\right) \leq 1$, so we have $f\left(\left\langle\pi_{1}(x), e_{\alpha_{j}}\right\rangle^{2}\right) \rightarrow 0$.

Define $P_{j}(x)=P(x)-\left\langle\pi_{1}(x), e_{\alpha_{j}}\right\rangle^{2} \in \mathcal{P}\left({ }^{2} H\right)$. Then $\left\|P_{j}\right\|=1$ and $\left|f\left(P_{j}\right)-1\right|=$ $\left|f\left(P_{j}-P\right)\right|=f\left(\left\langle\pi_{1}(x), e_{\alpha_{j}}\right\rangle^{2}\right) \rightarrow 0$. But

$$
\left\|P_{j}-P\right\|=\left\|\left\langle\pi_{1}(x), e_{\alpha_{j}}\right\rangle^{2}\right\|=\sup _{\|x\|=1}\left\langle\pi_{1}(x), e_{\alpha_{j}}\right\rangle^{2}=1,
$$

so we have a contradiction. Thus $\operatorname{sexp} B_{\mathcal{P}\left({ }^{2} H\right)}=\emptyset$.

Theorem 3. Let $H$ be non-separable real Hilbert space. Then the unit ball of $\mathcal{P}\left({ }^{2} H\right)$ contains no exposed points.

Proof. Let $P$ be an extreme point, so that

$$
P(x)=\sum_{\alpha \in A}\left\langle x, t_{\alpha}\right\rangle^{2}-\sum_{\beta \in B}\left\langle x, t_{\beta}\right\rangle^{2}
$$

relative to a suitably chosen orthonormal basis whose indexing set is the disjoint union $A \cup B$. Suppose that the functional $f$ exposes $P$. As in the proof of Theorem 2 it follows that $f\left(\left\langle x, e_{\alpha}\right\rangle^{2}\right)>0$ for every $\alpha \in A$ and similarly, $f\left(\left\langle x, e_{\alpha}\right\rangle^{2}\right)<0$ for every $\alpha \in B$. But

$$
\sum_{\alpha \in A} f\left(\left\langle x, t_{\alpha}\right\rangle^{2}\right)=f\left(\sum_{\alpha \in A}\left\langle x, t_{\alpha}\right\rangle^{2}\right) \leq 1,
$$

and hence $A$ must be countable. Similarly, $B$ is countable. Thus $H$ is separable. 


\section{ACKNOWLEDGEMENT}

The authors wish to thank the referee for pointing out Theorem 3 and several useful remarks.

\section{REFERENCES}

[1] C. Boyd and R.A. Ryan, Geometric theory of spaces of integral polynomials and symmetric tensor products, J. Funct. Anal. 179 (2001), 18-42. MR 2002b:46029

[2] Y.S. Choi, H. Ki and S.G. Kim, Extreme polynomials and multilinear forms on $l_{1}$, J. Math. Anal. Appl. 228 (1998), 467-482. MR 99k:46077b

[3] Y.S. Choi and S.G. Kim, The unit ball of $\mathcal{P}\left({ }^{2} l_{2}^{2}\right)$, Arch. Math. 71 (1998), 472-480. MR 2000c:46085

[4] Y.S. Choi and S.G. Kim, Exposed points of the unit balls of $\mathcal{P}\left({ }^{2} l_{p}^{2}\right)(p=1,2, \infty)$, (Preprint).

[5] S. Dineen, Extreme integral polynomials on a complex Banach space, (Preprint).

[6] B.C. Grecu, Extreme polynomials on Hilbert spaces, (Preprint).

[7] R.A. Ryan and B. Turett, Geometry of spaces of polynomials, J. Math. Anal. Appl. 221 (1998), 698-711. MR 99g:46015

Department of Mathematics, Kyungpook National University, Daegu, Korea (702701)

E-mail address: sgk317@knu.ac.kr

Department of Mathematics, Kyungpook National University, Daegu, Korea (702701)

E-mail address: sanghlee@knu.ac.kr 